\title{
Agôn
}

Revue des arts de la scène

5 | 2012

L'entrée en scène

\section{Pour un centième de seconde de virtuosité}

Performers, sportifs et danseurs dans l'arène

\section{Agathe Dumont}

\section{(2) OpenEdition}

Journals

Édition électronique

URL : http://journals.openedition.org/agon/2407

DOI : 10.4000/agon.2407

ISSN : 1961-8581

Éditeur

Association Agôn

\section{Référence électronique}

Agathe Dumont, "Pour un centième de seconde de virtuosité », Agôn [En ligne], 5 | 2012, mis en ligne le 25 janvier 2013, consulté le 20 avril 2019. URL : http://journals.openedition.org/agon/2407 ; DOI : 10.4000 /agon. 2407

Ce document a été généré automatiquement le 20 avril 2019.

Association Agôn et les auteurs des articles 


\title{
Pour un centième de seconde de virtuosité
}

\author{
Performers, sportifs et danseurs dans l'arène
}

\author{
Agathe Dumont
}

1 Été 2012. Stade Olympique. La dixième journée des jeux d'été de Londres se referme sur l'exploit du sprinter Usain Bolt, double champion olympique et recordman mondial du cent mètres. Quelques instants auparavant, rien n'était joué. Il aura fallu 9 secondes et 63 centièmes au champion pour entrer en scène et jouer le spectacle de sa victoire.

2 Ici, l'entrée en scène se confond avec le spectacle en lui même. Cependant, pour y parvenir, un rituel s'organise autour de l'athlète. D'un côté, l'entrée en scène la plus rapide du monde est savamment orchestrée par l'industrie du sport et des mass medias, caractérisée par une performance vécue comme un exploit, spectaculaire, aux confins de l'excellence technique. D'un autre côté, l'instant où le coureur s'apprête à partir exerce une étrange fascination sur le spectateur, entre maîtrise et incertitude ; dans ce moment où le corps se prépare mais où tout semble pouvoir basculer. Interprète hors-norme, Usain Bolt, dont la décontraction au départ est souvent relevée par les journalistes sportifs, est comparable à ceux que le sociologue Stéphane Héas qualifie d'«êtres exceptionnels ${ }^{1} »$.

3 Ce qui caractérise le sprinter est sa capacité à se jeter immédiatement dans l'action, sans transition. Ainsi, outre, le spectacle qui entoure la performance (clameur du stade, mise en scène de l'arrivée des athlètes, présentation), c'est l'instant où le coureur s'apprête à partir puis décolle qu'il faut observer: une puissante montée d'adrénaline, l'impact, le corps qui se déploie en un instant, la contraction et l'explosion des muscles. C'est au cours de ces quelques secondes de silence et d'immobilité suivies de quelques centièmes pendant lesquels les muscles explosent que l'athlète est le plus virtuose. Certes, on pourrait considérer comme virtuose ce qui est le plus spectaculaire : expertise technique, dépassement de soi, excellence (le geste sportif est objectivement mesurable), mais il existe une virtuosité plus invisible, un état de corps juste avant d'entrer en scène qui rompt avec tout code et suppose une toute autre organisation sensori-motrice pour le performer. En observant ce qui est mis en jeu au départ d'un sprint, il nous est possible de 
regarder autrement ce qui est mis en jeu avant la mise en mouvement du corps dans la danse ou dans d'autres formes spectaculaires. L'énergie mise en jeu, maximale dès les premiers centièmes de seconde, et la mise en tension du corps qui la précède rappellent ce que le sociologue du sport et des loisirs Paul Yonnet déclarait à propos du spectacle rock, un autre espace dans lequel une forme de virtuosité peut advenir en quelques centièmes de seconde :

La culture rock [...] ignore le crescendo, la montée progressive. On est dès le départ au plus fort du spectacle, au plus fort du feeling, ou peu s'en faut. Le rock ne donne pas à voir un dévoilement progressif de sa nature par segments s'additionnant les uns aux autres, son corps est là d'emblée tout entier. ${ }^{2}$

Dès lors, est-il possible de retrouver l'état de ce corps physiquement et cognitivement et «dès le départ au plus fort du spectacle» dans d'autres pratiques spectaculaires et performatives?

5 À partir des méthodes d'analyse du mouvement et d'analyse systémique du geste expressif ${ }^{3}$, nous proposons de décrypter cet état de corps à travers les exemples d'entrées en scène issues de spectacles de danse et dans lesquels nous retrouvons certaines des composantes de l'arène sportive ou de la scène rock. Plus précisément, nous nous intéresserons à ce qui se joue dans l'infiniment petit du muscle, le geste précis des performers juste avant le départ du mouvement, son intention et son attention ${ }^{4}$. Nous faisons donc référence au pré-mouvement tel que le définit Hubert Godard, soit l'élan, l'impulsion ou le starter du mouvement, la mise en tension des muscles et l'ajustement de la posture juste avant l'action. Ce pré-mouvement peut revêtir des qualités différentes, comme nous le verrons à travers les exemples de trois danseuses dont l'état de corps dans le mouvement dansé se rapproche, selon nous, de celui du sprinter. Un vocabulaire physique engagé laisse entrevoir un portrait de ces danseuses en athlètes : Emilie Sudre, Aurore Ponomarenko et Louise Lecavalier partagent un même goût pour la virtuosité.

Émilie Sudre, interprète hip hop, danse en 2005 Soli II d'Anthony Egéa, solo dans lequel l'entrée en scène deviendra pour elle une entrée dans l'arène. À la croisée de la danse et du sport, Aurore Ponomarenko, ancienne patineuse artistique, danse quant à elle dans $E$ ternelle Idole (2008), de la metteur en scène Gisèle Vienne, sur une patinoire. Enfin, Louise Lecavalier est une ancienne danseuse de la compagnie Lalala Human Steps ${ }^{5}$. En 1988, lors d'une tournée de la compagnie avec David Bowie, elle s'illustrera dans un véritable duo, entre danse et rock, avec le chanteur. Arène du break, ring de compétition, scène du rock : partant de l'hypothèse qu'une situation ou un espace conditionne le geste, nous envisagerons l'entrée dans l'action non pas comme le franchissement d'un seuil mais comme un saut dans le vide.

\section{À vos marques...}

7 L'espace dans lequel le corps entre en scène influe largement sur la construction du geste et sur la perception que nous en avons. À partir de ses recherches sur la construction physiologique de l'équilibre, Philippe Goudard, spécialiste du cirque, explique que différents dispositifs circulaires provoquent le même type de geste :

Spectacles du cirque ou de la corrida, concours d'athlétisme ou de gymnastique, match de rugby, football, hockey, combat sur un ring ou sur les pitts à coqs, courses de chevaux, cycliste ou de formule 1 , tournois de tennis, joutes d'improvisations théâtrales, jeux de dés... ont en effet des composantes communes. ${ }^{6}$ 
Et de poursuivre :

Le pratiquant montre, dans l'aire de jeu centrale, sa capacité à triompher du sort face au hasard, à contrôler la gravité face au déséquilibre, à maîtriser sa destinée devant l'incontrôlable. Il résout un projet moteur ou intellectuel faisant appel à une stratégie, une situation de confrontation volontaire au danger. ${ }^{7}$

9 De fait, au moment d'être dans les starting blocks, le corps du sprinter se tend, prêt à bondir dans l'espace, à la limite de l'action mais immobile. Le corps est en tension vers l'avant, mais le poids est également réparti entre l'appui en arrière pour garantir la propulsion et les trois appuis en avant (les deux mains portant le poids du buste), assurant une suspension du bassin, qui fait que le corps n'est pas figé dans une posture mais constamment dans une régulation tonique, pour maintenir cet équilibre précaire. Par empathie kinesthésique, cette posture procure au spectateur une sensation entremêlée de puissance et de vertige, créant instabilité et impermanence, principes moteurs et cognitifs qui animent le travail des performers dans les espaces à aire de jeu centrale. Cet état précis du corps, immergé dans l'arène va se retrouver dans l'entrée en scène de performers dans d'autres spectacles que nous proposons à présent de décrire.

\section{Émilie Sudre dans Soli II : l'arène du break}

Dans Soli II, pièce d'Anthony Egéa, le dispositif scénique est frontal et la zone d'action de la danseuse matérialisée au sol par un tapis blanc éclairé. Pourtant, une aire de jeu centrale telle que l'envisage Philippe Goudard est perceptible notamment à travers l'état de corps de la danseuse qui entame le spectacle par une longue marche de la soliste autour de cet espace. Les pas sont lents et réguliers, le dos est droit et le regard posé sur la ligne d'horizon ou clairement dirigé vers le public. Le sternum légèrement en avant, la danseuse a une démarche volontaire accentuée par le martèlement des chaussures à talons qu'elle porte. La tension entre la verticalité ou l'aplomb de la danseuse, le travail d'équilibre visible en raison de la hauteur des chaussures, et cet espace au sol est très palpable. Emilie Sudre semble délimiter son ring. Dès lors, cette entrée dans le silence ressemble à une entrée dans la "fosse aux lions", selon l'expression de la danseuse ${ }^{8}$. La posture est érigée, dynamique, le spectateur sent que le corps est prêt au mouvement. Pourtant, si le défi est bien présent, ce geste n'est pas assimilable à celui de l'arène sportive. La danseuse inverse les rapports de pouvoir propres au schéma compétitif puisque ce n'est pas tant l'espace qui influe sur son geste et procure cette sensation de tension extrême, mais elle qui délimite son espace en adoptant une marche géométrique et régulière ; c'est elle qui donnera le « coup de feu » de son propre départ. ${ }^{9}$

11 Les enjeux très particuliers du battle hip hop, entre prouesse et rituel, nous reviennent en mémoire face à ce spectacle ; dans le battle l'état de corps des performers naît de la fusion, dès l'instant où le corps s'élance, de la figure de l'athlète et de la figure de l'artiste. Là encore, pas de crescendo, pas de transition, le corps doit être immédiatement performatif, comme le corps du rocker, comme le corps du sprinter. Or, c'est justement ce dernier élément que le philosophe Michel Bernard analyse comme relevant d'un modèle normatif voire coercitif, et actif dans la corporéité sportive. Michel Bernard parle alors d'exhibition du corps dans le spectacle sportif car la virtuosité y est manifeste et renforcée par sa médiatisation :

Le spectacle sportif n'est pas, et ne se veut pas, en effet, la représentation pour un public d'une histoire singulière d'événements fictifs ou vrais, en quelque sorte le 
simulacre d'un conflit contingent passé ou imaginaire; simulacre préparé et élaboré pour satisfaire la curiosité et, si possible, provoquer des émotions. C'est, bien au contraire, la réalité présente, effective qui est exhibée: celle d'une confrontation inter-individuelle ou intergroupale universellement et rigoureusement codée, contrôlée et jugée. ${ }^{10}$

En ce sens, la virtuosité est considérée comme opérante dans le show sportif tel que le décrivent Georges Vigarello ou Paul Yonnet: sujet des mass medias, fer-de-lance du spectacle de masse, agent principal de la transformation du sportif en héros ${ }^{11}$. À l'inverse, la corporéité dansante, telle qu'elle se construit dans les arts du spectacle serait déconstruction, le performer y joue différemment son entrée dans l'arène. Un exemple comme cette entrée en scène d'Emilie Sudre dans Soli II, cette mise en tension avant l'action pose problème car elle semble précisément relever des deux modèles en même temps. Michel Bernard, d'ailleurs, nuance son propos en distinguant différentes pratiques sportives dans lesquelles la théâtralisation du geste et de l'action n'est pas la même, répondant à des finalités variables, par exemple la part "artistique » de certains sports tels que la gymnastique ou le patinage artistique même s'ils restent "rigoureusement codés, contrôlés et jugés ", réflexion que nous pourrions donc étendre à la qualité de geste développé dans les battles et que l'on retrouve ici, performé autrement, dans la danse d'Emilie Sudre. Il s'agit donc d'un état de corps assimilable à la tension dramatique au moment de servir pour un match de tennis à l'issue inconnue ou la concentration du skieur derrière le portillon d'une descente tout autant qu'à l'engagement du danseur qui va s'élancer sur le plateau. La virtuosité mise en jeu répond alors à une urgence, le performer n'a plus le choix. Peu importe la performance, à ce moment précis le corps se tend et bascule vers le départ. Il est au seuil d'un déséquilibre qu'il maintient de toutes ses forces, une virtuosité silencieuse et presque invisible qui nous fait percevoir qu'il ou elle est disposé(e) à agir.

\section{Prêts ?}

Dès qu'il est prêt au départ, le corps est déjà dans l'action. Le sprinter s'élève sur ses appuis, prêt à se jeter dans le vide. Dans la théorie labanienne, socle de l'analyse du mouvement, l'idée d'effort est centrale pour comprendre ce moment juste avant l'action. Effort, chez Laban, est l'un des paramètres essentiels du mouvement dansé, une manifestation physique de l'acte (on parle parfois de qualité du mouvement), «chaque effort est initié par une disposition à agir ${ }^{12}$ ». Le terme anglais est à comprendre comme l'entrelacement de la projection (fictionnelle) du geste dans l'espace et de son déroulement, dans un effort soutenu. Cette disposition à agir se rattache à la phase d' attention du mouvement et décrit donc l'état tonique du danseur, son impulsion, ce qu'Hubert Godard analyse donc comme le pré-mouvement ${ }^{13}$.

14 À travers l'effort, Laban décrit le mouvement par des verbes d'action (dites actions " dynamiques ou élémentaires ») repérables dans la plupart des gestes quotidiens. Chaque verbe d'action est associé à une sensation motrice ${ }^{14}$ et met en jeu les quatre paramètres de l'effort: le poids (Strong/ferme et Light/léger), l'espace (Direct/direct et Flexible/ flexible), le temps (Quick/soudain et Sustained/soutenu) et le flux (Bound/contrôlé et Free/ libre). Les deux verbes fondateurs sont «Punching/ Frapper » et «Floating/ Flotter ». À partir de ces paramètres, Laban définit huit actions ou intentions (qui sont aussi des perceptions du geste) élémentaires, allant du maximum d'effort au minimum. Frapper ou 
«lutter contre» (struggling against) et flotter ou «attitude d'abandon» (indulging in) représentent les deux extrêmes. La palette de l'effort se décline ainsi en huit combinaisons entre ces deux pôles. L'effort est donc avant tout un principe qualitatif qui se dessine dans la part « invisible » du geste : sa tessiture, son impulsion. Il détermine l'état tonique du performer. L'effort au départ du sprint est ainsi caractérisé par la double-tension entre struggling against et indulging in, caractéristique d'une immobilité qui cache une tension musculaire extrême. Il s'agit donc bien d'une intention de partir ou de surgir, non suivie d'action mais dont l'attention est palpable. Le spectateur peut aisément projeter le mouvement à venir, tant la tension musculaire et la posture du corps tendent vers le départ.

\section{Aurore Ponomarenko : métamorphoses du geste sportif dans Éternelle Idole}

On retrouve cet état de corps dans Éternelle Idole ${ }^{15}$, solo pour une patineuse, mise en scène par Gisèle Vienne. Inspirée par l'imagerie et les fantasmes liés à la pratique de ce sport (la patinoire, le costume de la patineuse, etc.) la chorégraphe ne conserve pourtant du geste patiné que la tension musculaire avant le départ. Supprimant tout enjeu de performance, elle donnera à voir la suspension maximale avant l'action plus que l'action elle même, densifiant cet état, puisant au plus profond des ressources motrices et cognitives d'Aurore Ponomarenko, la patineuse. Cette dernière commence la pièce par une succession de poses immobiles. Le mouvement n'a pas commencé, mais la lutte des muscles est déjà là, le corps se prépare : "La partie hyper lente, explique-t-elle, on ne la fait jamais et c'est ce qui demande le plus d'effort, c'est la partie où les muscles sont le plus en tension. Alors que visuellement, ce n'est pas ce qui est le plus performant. » En effet, chaque mouvement est décomposé à l'extrême. Pieds croisés, sternum et regard vers le ciel, bras en croix sur la poitrine, pirouette et port de bras au ralenti, lente extension vers une arabesque, posture de révérence arrêtée ou simplement posture debout, verticale, seule au milieu de la patinoire. De même, à la fin du spectacle, une scène de compétition est jouée. Présence de l'entraineur, clameur de la patinoire, programme ; le geste est conditionné par cet environnement compétitif qui suppose une présence (en tension), un geste (engagé). Sur le bord de la patinoire, la patineuse se tient immobile, prête à basculer dans l'arène, les muscles arc-boutés. Rien ne bouge, pourtant tout bouge. Le spectateur perçoit déjà les triples axels tout comme il voit le sprinter franchir la ligne avant même que le troisième ordre ne soit donné. S'il n'est pas question de «jouer» la compétition, il semble bien que seul un corps athlétique et rompu à l'exercice soit à même de provoquer chez le spectateur la sensation à ce point invisible d'un corps dans les starting blocks, et qui capte son attention avant même de se mouvoir. Cet état est aussi celui que nous renvoie kinesthésiquement le joueur de football avant de tirer un penalty, l'immobilité du joueur de rugby face aux barres, lorsque son regard se pose à l'horizon et qu'il visualise l'action qui va suivre ; une virtuosité silencieuse juste avant l'événement. On retrouve alors dans Éternelle Idole le départ avant l'action, l'instant d'immobilité avant l'explosion, ce qu'André Lepecki décrit comme une "vibration du microscopique ${ }^{16} »$. 


\section{Partez !}

16 À la seconde où le coup de feu signalant le départ retentit, le coureur relâche ses appuis. Dès les premières percussions des pieds au sol, la posture se structure. La jambe avant se replace petit à petit près de la projection verticale du bassin puis devant celle-ci afin d'effectuer un redressement complet du buste. Malgré sa complexité, l'ensemble de ces actions se déroule en quelques centièmes de seconde. Car, à peine entré dans la course, l'athlète franchit la ligne. La progression de l'action est donc presque invisible pour un œil non aguerri. Le crescendo est rapide et sans paliers. Nous sommes donc immédiatement «au plus fort de l'action» (Yonnet). Le fait que le corps bascule si soudainement dans l'action est là encore vecteur de virtuosité. Le performer rompt les fils qui retiennent le déséquilibre pour bondir. Les réflexions de Paul Yonnet sur le rock auxquelles nous nous sommes référée nous conduisent à envisager un rapprochement entre la posture (et l'organisation sensori-motrice) de l'athlète et de la rockstar. ${ }^{17}$

La spécificité du spectacle rock est qu'il met en scène un corps hors norme, un geste nourri de fantasmes de puissance, non éloigné donc des corps athlétique qui semblent parfois défier les limites du corps humain. L'engagement physique, l'exposition de soi, en même temps que la maîtrise technique, jusque dans les infimes tissus du corps, sont palpables à plusieurs niveaux. Tant dans la mise en jeu de leur corps que dans progression dramatique de leur geste, l'athlète et la rockstar partagent une même virtuosité dès la seconde qui précède le mouvement. Cette virtuosité-ci ne relève pas tant d'une maitrise technique que d'une faculté à s'exposer sans médiation. Une fois encore, le spectacle vivant est riche d'exemples qui mettent en scène cette même capacité d'exposition lorsque les performers s'élancent sur le plateau.

\section{Louise Lecavalier et David Bowie : l'arène du rock}

Nous prendrons ici un exemple qui met en scène une danseuse-athlète, Louise Lecavalier et une rockstar, David Bowie, lors de la tournée mondiale de ce dernier en 1988. Les duos entre la danseuse phare de la compagnie Lalala Human Steps et le chanteur sont nombreux et nous proposons de nous intéresser au premier d'entre eux, alors que les deux performers entrent en scène sur l'introduction de la chanson Look Back in Anger. Face à face, ils se toisent, ce qui correspondrait à une phase de mise en tension des muscles, d' attention. À plusieurs reprises, Louise Lecavalier, s'éloigne puis revient vers le chanteur, s'arrête, puis soudainement, la danseuse repart à pleine vitesse ; le départ est donné. Dans une interview donnée à la télévision canadienne en 1988, Louise Lecavalier évoque une sensation éprouvée à travers le concert rock dont elle dit que c'est ainsi qu'elle voulait danser. De la même manière, David Bowie raconte que les deux corps des performers sont à tel point liés par la même grammaire physique, qu'on ne peut lire la musique qu'à travers le mouvement. Expression de la puissance, érotisation du geste, mise en scène d'une fascination pour le corps, pour ses limites voire pour sa mise à mort ${ }^{18}$, les premiers gestes de Louise Lecavalier, la tension qu'elle installe dans tout son corps, prêt à bondir à tout instant, trouve toute sa mesure dans la syntaxe particulière du corps de la rock star. Dans cette danse comme dans la musique, il n'existe aucune transition. Ce que nous voyons en scène dans le duo David Bowie/Louise Lecavalier, ce que nous éprouvons par empathie kinesthésique, est une incandescence du geste. Le geste existe par et pour lui-même, 
immédiatement dans l'action, devançant la prise de parole du chanteur ; à pleins pouvoirs et à pleine vitesse, il surgit dès les premières secondes des accords de guitare. ${ }^{19}$

Ce geste, rapide, intense, est donc à l'image de ce qu'est le rock et l'on pourrait aisément relier la puissance de ce corps immédiatement performant et performatif dont parle Paul Yonnet à ce que décrit Dan Graham du débit vocal du leader du groupe The Kinks ${ }^{20}$, dans un ouvrage majeur sur le rock:

Ray Davies avait un pas d'avance sur le phrasé incroyable de Little Richard - sa propension à étirer un point jusqu'à la ligne en haletant et à nous asphyxier; en quoi le problème mécanique du souffle (inspiration) et de l'articulation (expiration) aide à déterminer la forme. [...] Ici tout est comme précipité, arrêté et repris, puis une respiration, monotone et fragile - susceptible de se casser et de se recomposer à tout moment. [...] Emballés, superficiels, à la recherche d'une bouffée d'oxygène condensée en agoraphobie. L'émotion agoraphobique atteint le vide parfait dans un vortex sans fond d'émotion perdue, nostalgique et douce, dans l'absence d'apogée insipide, tour à tour non modulée ou sur-modulée [...]. ${ }^{21}$

Autant de qualificatifs d'une corporéité soumise à l'urgence de son geste, d'un corps qui fait acte de puissance, qui font écho à la typologie qu'Ann Cooper Albright dresse en 1997 à propos des corps virtuoses, illustrant notamment son propos par l'exemple de Louise Lecavalier :

Hyper athlétique, de courts moments de repos, s'accrochant ou tirant, unité discontinue, physicalité audacieuse, suffisamment serrée et suffisamment forte, une danse féroce, bagarreuse, puissante mais jamais contenue, des mouvements précis et dirigés, chaotique, qualité exponentielle. ${ }^{22}$

21 Une tension extrême est donc présente dans l'état de corps de ces puissantes entrées en scène, combinée à une fragilité qui donne la sensation que le performer est sans cesse « sur le fil » afin que, jamais, le spectateur ne puisse reprendre son souffle.

\section{«Walk the line »}

L'idée d'un geste virtuose comme le lieu du dépassement d'un code et de la surexposition du corps se dessine dans les exemples de performers décrits ici. Ce geste virtuose que l'on perçoit dans le laps de temps qui précède l'action n'est pas une fin en soi, c'est un geste irrésolu : «Dans le rock, en effet, s'il y a de l'intro, il n'y a pas de chute, pas d'aboutissement. Le rock n'a pas la science des fins, auxquelles il se résout difficilement. » ${ }^{23}$ S'il n'y a pas d'aboutissement, ce qui caractérise cette virtuosité dans le corps de la rock star comme dans celui des athlètes ou des danseurs qui s'en approchent, est l'épuisement. Cette virtuosité est l'affaire de quelques centièmes de seconde et ne se lit que dans l'infiniment petit de la tension musculaire, de l'attention et de l'intention avant même qu'il ou elle ne commence à se mouvoir. Les uns comme les autres n'ont d'autre choix que d'être immédiatement dans l'action. Les quelques secondes au départ d'un sprint sont les seules dont le sprinter peut s'emparer pour entrer dans l'arène ; quelques secondes plus tard, le spectacle est fini. Les performers que nous évoquons ici font l'expérience de ce même état de corps. Si la temporalité du spectacle vivant n'est évidemment pas la même que celle d'une course olympique, ce qui précède la mise en jeu du mouvement moteur est semblable en termes d'énergie et de tension musculaire. Le performer, conscient au creux de chacun de ses muscles qu'il n'a d'autre choix que de se jeter immédiatement dans le mouvement, refuse toute phase introductive ou temps d'attente qui viendraient justement défaire ce que corps en tension s'efforce d'engranger pendant ce pré- 
mouvement. Pour le spectateur comme pour le performer, la virtuosité - qu'elle soit attendue ou non - ne tolère pas un millième de seconde d'hésitation, elle est donc bien un saut dans le vide.

Pour conclure notre réflexion, il convient de nuancer le rapport entre spectacle sportif et spectacle artistique, car la finalité du geste virtuose n'y est pas la même. L'entrée en scène avortée de l'athlète chinois Liu Xiang lors de la finale du 110 mètres/haies des même jeux olympiques d'été 2012 en est un parfait exemple. Alors que son corps est prêt à bondir, la posture de départ tendue vers la ligne d'arrivée, l'athlète est foudroyé dès ses premières foulées par un claquage musculaire qui le force à interrompre sa course. La rupture est aussi immédiate et explosive que le fut le départ ; le spectacle est terminé, la virtuosité sportive ne tolère pas l'échec. Mais, sur la scène du spectacle vivant, il en est autrement. Le corps de la rock star, celui du danseur, sont, dès l'entrée en scène, des corps que Bettina Brandl-Risi définit au théâtre comme « Failure as a chance " ${ }^{24}$, l'échec comme chance. Le luxe du virtuose est finalement de pouvoir échapper au sens, envisageable autant du point de vue de la maitrise absolue que de celui de l'imperfection totale. Ainsi, le pré-mouvement, la tension du corps avant l'action nous renseigne sur cette différence fondamentale. La posture de l'athlète dans le stade n'est que tournée vers l'hyper performance, ce que Bettina Brandl-Risi nomme «virtuosity as outperforming ${ }^{25}$. Les postures de David Bowie, de Louise Lecavalier, d'Aurore Ponomarenko ou d'Emilie Sudre sont bien plus indisciplinées. Plus que la maitrise absolue, elles renvoient au spectateur la possibilité de faillir, le déséquilibre. Si leur but semble être le même - exploser à la seconde même où le corps entre en scène - avant même d'agir, le projet de l'athlète comme de l'artiste est incorporé dans sa posture. Le poids, l'équilibre, l'organisation verticale nous renseignent sur sa "disposition à agir » (Laban). Une fois les barrières de l'arène franchies, le saut dans le vide dépend de l'intention de chacun; esthétique, sportive, elle déterminera la finalité ou la non-finalité du geste virtuose ainsi engagé.

\section{BIBLIOGRAPHIE}

Bernard, Michel. « Les paradoxes du spectacle sportif ou les ambiguïtés de la compétition théâtralisée ». In. Sport et Société. Approche socio-culturelle des pratiques. Paris : Vigot, 1981.

Brandl-Risi, Bettina. « The new virtuosity. Outperforming and Imperfection on German Stage ». Theater. $\mathrm{N}^{\circ}$ 37, vol. I, 2007. P. 9-37.

Brandstettern Gabriele. Szenen des Virtuosen. Berlin: Transcript Verlag, 2012.

Cooper-Albright, Ann. Choreographing Differences. Middletown : Weyselan University Press, 1997.

Godard, Hubert. « Le Geste et sa perception ». In. La danse au XX ${ }^{e}$ siècle. Paris : Larousse, 1995. P. 235-241.

Goudard, Philippe. Le Cirque entre l'élan et la chute. Saint-Gély-du-Fesc : Editions Espaces 34, 2010. Graham, Dan. Rock/music. Textes. Traduit de l'américain par Vincent Pécoil. Dijon : Les Presses du Réel, 1999. 
Héas, Stéphane. Les Virtuoses du corps. Enquête sur des êtres d'exception. Paris : Max Milo, 2010.

Laban, Rudolf. La Maîtrise du mouvement. Traduction française de Jacqueline Challet-Haas et Marion Bastien. Arles : Actes Sud, 2007.

Roquet, Christine. Fattoumi-Lamoureux, danser l'autre. Paris : Séguier, 2009.

Vigarello, Georges. Du jeu ancien au show sportif : La Naissance d'un mythe. Paris : Seuil, 2002.

Yonnet, Paul. « L'esthétique rock ». Le Débat. N 40, mai-septembre 1986. P. 58-64.

Yonnet, Paul. Huit leçons sur le sport. Paris : Gallimard, 2004.

\section{NOTES}

1. Héas, Stéphane. Les Virtuoses du corps. Enquête sur des êtres d'exception. Paris : Max Milo Editions, 2010.

2. Yonnet, Paul. « L'esthétique rock », Le Débat. n 40, mai-septembre 1986. P. 63.

3. Nous distinguons ici l'analyse du mouvement qui prend en compte des paramètres physiologiques et biomécaniques de l'analyse expressive du geste qui s'interroge sur la portée symbolique et expressive du geste, compris comme une totalité et non comme une unité fonctionnelle du corps. Voir: Roquet, Christine. "Postface». In : Fattoumi-Lamoureux, danser l'autre. Paris : Séguier, 2009.

4. Pour Laban, pédagogue et théoricien du mouvement du début du siècle, il existe quatre étapes dans le mouvement : attention, intention, décision, précision. Sa théorie eukinétique donne les bases de l'analyse du mouvement telle qu'elle s'est développée au cours du vingtième siècle. Avec l'analyse systémique du geste expressif, Hubert Godard étudie ces phases préparatoires en développant le concept de pré-mouvement ou d'anacrouse gestuelle. Il définit l'état tonique du danseur et son rapport à la gravité, induisant une qualité spécifique à chaque geste, à chaque danseur en fonction de son état. C'est par ailleurs cet état qui détermine le rapport du danseur à son environnement, au monde, et rend ainsi son geste expressif. Voir : Godard, Hubert. « Le geste et sa perception ». In: La Danse au XXe siècle. Paris :Larousse, 1995. P. 235-241.

5. Louise Lecavalier travaille aujourd'hui avec sa propre compagnie Fou Glorieux à Montréal après avoir quitté Lalala Human Steps en 1997.

6. Goudard, Philippe. Le Cirque entre l'élan et la chute. Saint-Gély-du-Fesc: Editions Espaces 34, 2010. P. 40.

7. Id. p. 52.

8. Sauf mention contraire, les citations des interprètes sont issues d'un travail de terrain mené entre 2006 et 2011 dans le cadre d'un doctorat en arts du spectacle.

9. Anthony Egéa, Soli II, interprète Emilie Sudre, création les 3, 4, 5 novembre 2005 : Festival Novart - Glob théâtre - Bordeaux. http://www.youtube.com/watch? v=It8Nnvh2wjE\&feature=plcp, page consultée le 26/12/2012.

10. Bernard, Michel. «Les paradoxes du spectacle sportif ou les ambiguïtés de la compétition théâtralisée ». In Sport et société. Approche socio-culturelle des pratiques. Paris,Vigot : 1981. P. 355. (Souligné dans le texte).

11. Voir : Vigarello, Georges. Du jeu ancien au show sportif : La Naissance d'un mythe. Paris : Seuil, 2002. Yonnet, Paul. Huit leçons sur le sport. Paris : Gallimard, 2004.

12. Laban, Rudolf. La Maîtrise du mouvement. Traduction française de Jacqueline Challet-Haas et Marion Bastien. Arles : Actes Sud, 2007. P. 237. Le terme employé en anglais est celui de Effort. (Nous soulignons) 
13. Pour Laban, il existe quatre étapes dans le mouvement: attention, intention, décision, précision. En construisant une analyse systémique du geste expressif, Hubert Godard analyse ces phases préparatoires, sur l'impulsion en développant le concept de pré-mouvement ou d'anacrouse gestuelle. Il définit l'état tonique du danseur et son rapport à la gravité, induisant une qualité spécifique à chaque geste, à chaque danseur en fonction de son état. C'est par ailleurs cet état qui détermine le rapport du danseur à son environnement, au monde, et rend ainsi son geste expressif.

14. Cette scission entre action et perception pose d'ailleurs un problème conceptuel et un problème de traduction. En effet, Laban décrit ces termes comme des « sensations motrices ». En utilisant la forme continue [-ing] en anglais, action et perception sont confondues. Tandis que l'utilisation des verbes à l'infinitif en français ne prend en compte que l'action, au détriment de la sensation.

15. Gisèle Vienne, Eternelle Idole, interprète Aurore Ponomarenko, création les 27 et 28 février 2009, Festival Antipodes'09, Le Quartz - Scène Nationale de Brest. http://www.g-v.fr/fr/shows/ eternelle-idole. Page consultée le 19/09/2017.

16. Lepecki, André. "Still: On the vibratile Microscopy of Dance ». In. Remebering the body. Vienne : Catalogue de l'exposition STRESS de l'Austrian Museum of the Applied Arts, 2000. P. 360.

17. En effet, il ne s'agit pas d'analyser le vocabulaire gestuel mais la manière dont le corps s'organise, d'un point de vue proprioceptif et moteur, pour s'engager dans le mouvement.

18. Le 3 juillet 1972 David Bowie met à mort son double scénique Ziggy Stardust lors du « Rock n' roll suicide concert » à L'Hammersmith Odeon de Londres.

19. Look Back in anger tournée de la compagnie Lalala Human Steps avec le chanteur David Bowie, interprètes David Bowie et Louise Lecavalier, 1988. http://www.youtube.com/watch? $\mathrm{v}=$ YcaH4kdGU, page consultée le 26/12/2012.

20. Groupe de rock anglais formé en 1964 par Ray et Dave Davies.

21. Graham, Dan. Rock/music. Textes. Traduit de l'américain par Vincent Pécoil. Dijon: Les Presses du Réel, 1999. P. 16.

22. "Hyper athletic, brief moments of stillness, grabbing or pulling, discontinuous unity, physical audacity, tight enough and strong enough, fierce dancing, rough-and-tumble, strong but not contained, tight and driven movements, chaotic, expansive quality. » Cooper-Albright, Ann. Choreographing Differences. Weyselan University Press: Middletown, 1997. P. 28 et suivantes. (Nous traduisons)

23. Yonnet, Paul, art.cit., p. 70.

24. Brandl-Risi, Bettina. "The new virtuosity. Outperforming and Imperfection on German Stage ». Theater. $\mathrm{N}^{\circ}$ 37, vol. I, 2007. P. 9-37.

25. Ibid. L'idée de proposer une corporéité spécifique au virtuose émane du projet proposé par Gabriele Brandstetter : "To investigate the transgressive model of performance offererd by the virtuoso's stage " / "Enquêter sur les modèles transgressifs offerts par la scène virtuose ». Brandstetter, Gabriele. « The Virtuoso's Stage: A Theatrical Topos ». Theater Research International. Vol. 32, n² 2, 2007. P. 183. Les travaux du groupe sont publiés en allemand dans: Brandstetter, Gabriele. Szenen des Virtuosen. Berlin: Transcript Verlag, 2012. 


\section{RÉSUMÉS}

Le spectacle sportif et le spectacle vivant ont parfois des composantes communes. L'espace, le geste performé, les états de corps mis en jeu se retrouvent de l'arène à la scène. À partir des techniques d'analyse du mouvement, nous proposons d'explorer le geste qui précède l'action du corps, son "pré-mouvement» (Hubert Godard) en comparant le geste sportif au geste chorégraphique. Nous nous intéresserons donc aux quelques secondes qui précèdent l'entrée en action dans lesquelles se joue une virtuosité presque invisible aux yeux du spectateur, mais perceptible dans le corps du performer

INDEX

Mots-clés : danse, performance, sport, corps, geste, mouvement, technique

\section{AUTEUR}

\section{AGATHE DUMONT}

Agathe Dumont est docteure de l'université Sorbonne-Nouvelle Paris 3 et enseigne la danse en arts du spectacle à l'université Lumière-Lyon 2 et en STAPS à l'université Paris-Ouest Nanterre. agathe.dumont[at]gmail.com 